\section{Actividad física y cáncer de mama: un tratamiento dirigido}

\author{
KAROL RAMÍREZ ${ }^{1, a}$, FRANCISCO ACEVEDO², MARÍA ELISA HERRERA², \\ CAROLINA IBÁÑEZ ${ }^{2}$, CÉSAR SÁNCHEZ ${ }^{2}$
}

\section{Physical activity and breast cancer}

In Chile breast cancer $(B C)$ is the first cause of death in women. While the most important risk factor for its development is estrogenic stimulation, environmental factors and lifestyles also contribute to its pathogenesis. Epidemiological studies show a direct relationship between physical activity (PA), incidence and recurrence of $B C$. Supervised $P A$ practice is recommended in most cancer patients to improve their quality of life, to reduce adverse effects from treatment and eventually to improve the prognosis of the disease. We review the epidemiological evidence linking $P A$ and $B C$ and the biological basis of this relationship. We also review the relevant interventional studies and we explore some practical indications of $P A$ in patients with $B C$, as a model for other tumors of epidemiological importance.

(Rev Med Chile 2017; 145: 75-84)

Key words: Breast Neoplasms; Exercise; Secondary Prevention; Neoadjuvant Therapy; Neoplasms, Second Primary.
${ }^{1}$ Carrera de Kinesiología, UDA Ciencias de la Salud, Pontificia Universidad Católica de Chile. ${ }^{2}$ Departamento de HematologíaOncología, Facultad de Medicina Pontificia Universidad Católica de Chile. Santiago, Chile. ¿Licenciada en Kinesiología. MBAC en Salud.

Recibido el 10 de diciembre de 2015, aceptado el 22 de junio de 2016.

Correspondencia: César Sánchez Departamento de HematologíaOncología, Pontificia Universidad Católica de Chile.

Diagonal Paraguay 319, Santiago. Teléfono: 56-2-23546900

Fax: 56-2-22472327

csanchez@med.puc.cl
$\mathrm{E}$ 1 cáncer es una de las primeras causas de muerte en el mundo ${ }^{1}$. En Chile es la segunda causa de mortalidad ${ }^{2}$. La mayoría de los factores de riesgo para su desarrollo son ambientales y dependientes de estilos de vida; solo en $5-10 \%$ de los casos es pesquisada una etiología genética-hereditaria ${ }^{3}$. De allí, la importancia de controlar la exposición a agentes cancerígenos, y modificar conductas que disminuyan el riesgo de cáncer y la carcinogénesis ${ }^{1}$. La carcinogénesis es un proceso de multipasos a través del cual una célula normal se transforma en neoplásica, habitualmente en décadas ${ }^{4}$. Durante este proceso, la célula normal adquiere características distintivas: proliferación sostenida y autónoma, resistencia a la muerte celular, evasión de la respuesta inmune, alteración del metabolismo energético, generación de un ambiente inflamatorio e inestabilidad de su material genético. Esto le confiere ventajas comparativas sobre la célula normal y sirve al proceso de iniciación, promoción y progresión de la enfermedad ${ }^{5}$.

La detección temprana y avances en los trata- mientos oncológicos han mejorado el pronóstico y la sobrevida de los pacientes con cáncer. De manera global, se estima que la tasa de sobrevida libre de enfermedad a los 5 años es alrededor de $60 \%$. Sin embargo, para algunos tipos de cáncer detectados precozmente, la tasa de sobrevida puede ser superior a 90\%, como es el caso del cáncer de mama $(\mathrm{CM})^{6}$.

Según la Organización Mundial de la Salud (OMS), existen 25 millones de personas en el mundo que son sobrevivientes de cáncer ${ }^{7}$. La mayoría de ellos experimentan efectos secundarios derivados de la enfermedad y sus tratamientos, los que pueden perdurar en el tiempo, incluso una vez finalizada la terapia antineoplásica ${ }^{2}$.

Junto con mejorar la adherencia a tratamientos quimiopreventivos de largo plazo (por ejemplo, terapia endocrina en cáncer de mama), cambios en estilos de vida, la actividad física (AF) dirigida dentro de ellos, son estrategias relevantes de prevención secundaria ${ }^{8,9}$. Dada la creciente evidencia del rol de la AF en cáncer, el objetivo de este artículo es revisar los resultados de la literatura 
recientemente publicada sobre el efecto de la $\mathrm{AF}$ en la prevención, tratamiento y seguimiento de pacientes con CM, y generar recomendaciones basadas en esta evidencia.

\section{Metodología}

Se consultaron las bases de datos Pubmed Central, EMBASE y OVID durante los meses de mayo-octubre de 2015. La estrategia de búsqueda, combinando el uso de operadores booleanos AND y OR, fue la siguiente: breast neoplasm, physical activity, exercise, aerobic, resistance, chemotherapy, survivor. Se incluyeron en esta revisión, estudios que evaluaron el efecto de la AF en pacientes con $\mathrm{CM}$ en prevención, durante tratamiento antineoplásico y sobrevivientes.

\section{Cáncer de mama}

El CM es una enfermedad heterogénea que consta de varios subtipos ${ }^{3}$. El 75-80\% de las neoplasias de mama son dependientes del estímulo estrogénico, por tanto, tratamientos que regulan la actividad estrogénica son fundamentales en su manejo ${ }^{4}$. Resumidamente, el tratamiento curativo del CM puede involucrar tratamientos localizados que incluyen 1) cirugía y 2) radioterapia; y tratamientos sistémicos: 3) terapia endocrina, basada en el uso de moduladores del receptor de estrógeno (tamoxifeno), inhibidores de la aromatasa, supresión de la función ovárica, y sus combinaciones. Estos últimos están asociados a efectos colaterales inducidos por la deprivación y modulación estrogénica crónica (5 a 10 años en la terapia endocrina oral) $)^{3}$; 4) Quimioterapia, consistente en el uso de drogas citotóxicas para dañar el material genético de las células neoplásicas e impedir su replicación. No es selectiva, es decir, que no sólo afecta a células tumorales, sino que también a células normales, especialmente aquellas de rápido crecimiento, como las de cabello, piel, uñas, mucosas y glóbulos blancos, entre otras, lo cual produce efectos adversos no deseados en el tejido normal. Riesgos relevantes de algunas drogas citotóxicas son la cardiotoxicidad, la infertilidad y menopausia $\operatorname{precoz}^{3,5}$ y 5) Anticuerpos monoclonales. Se prescriben hasta por un año $y$, mientras son generalmente bien tolerados, existe un riesgo, inferior a $5 \%$, de falla cardiaca subclínica determinada por ecocardiograma $\mathrm{o}$, menos frecuentemente, clínicamente significativa ${ }^{3,10}$ (Tabla 1 ).

\section{Efectos secundarios del tratamiento del CM}

Las intervenciones terapéuticas pueden causar efectos secundarios agudos o a largo plazo, que tienden a disminuir la capacidad funcional y reducir la calidad de vida de los pacientes con cáncer ${ }^{2,5,6,11}$ (Tabla 2).

El $80-96 \%$ de las pacientes con CM presentan fatiga durante la quimioterapia y alrededor de un tercio reporta que esta persiste meses, e incluso años, luego de finalizado el tratamiento sistémi$\mathrm{Co}^{12}$. La fatiga interfiere con el funcionamiento habitual del paciente, generando una disminución de los niveles de AF durante el periodo de tratamiento, incluso, una vez finalizado sus tratamientos, se ha demostrado que persisten con niveles de AF disminuidos, a veces menores a los que tenían previo al diagnóstico ${ }^{12-15}$.

Tabla 1. Tipos de tratamiento en cáncer de mama, con intención curativa y sus objetivos

\begin{tabular}{|ll|}
\hline Tipos de tratamientos & Objetivos \\
Localizados & Remover el tumor primario y conocer el estado de los linfonodos axilares \\
\hline $\begin{array}{l}\text { 1. Cirugía } \\
\text { 2. Radioterapia }\end{array}$ & Controlar enfermedad micro-metastásica residual en la mama/pared torácica y axila \\
\hline Sistémicos & Control de enfermedad micro-metastásica local, regional y sistémica hormono dependiente \\
\hline $\begin{array}{l}\text { 3. Terapia endocrina } \\
\text { 4. Quimioterapia }\end{array}$ & Control de enfermedad micro-metastásica local, regional y sistémica \\
\hline \begin{tabular}{l} 
5. Anticuerpos monoclonales \\
Bloquean acción del receptor del factor de crecimiento epidérmico tipo 2, el cual se so- \\
\hline
\end{tabular} \\
\hline
\end{tabular}


Tabla 2. Signos y síntomas asociados a los tratamientos antineoplásicos utilizados en cáncer de mama localizado

\begin{tabular}{|c|c|c|c|c|c|}
\hline & Cirugía & Quimioterapia & Radioterapia & $\begin{array}{c}\text { Terapia } \\
\text { hormonal }\end{array}$ & $\begin{array}{c}\text { Terapia } \\
\text { biológica }\end{array}$ \\
\hline Fatiga & $\sqrt{ }$ & $\sqrt{ }$ & $\sqrt{ }$ & $\sqrt{ }$ & $\sqrt{ }$ \\
\hline Dolor & $\sqrt{ }$ & $\sqrt{ }$ & $\sqrt{ }$ & $\sqrt{ }$ & \\
\hline Cardiotoxicidad & & $\sqrt{ }$ & $\checkmark$ & $\sqrt{ }$ & $\checkmark$ \\
\hline Toxicidad pulmonar & & $\sqrt{ }$ & $\sqrt{ }$ & & \\
\hline Neuropatía periférica & $\sqrt{ }$ & $\sqrt{ }$ & $\sqrt{ }$ & & \\
\hline Cambios cognitivos & $\sqrt{ }$ & $\sqrt{ }$ & & $\checkmark$ & \\
\hline Infertilidad/menopausia precoz & & $\sqrt{ }$ & & $\sqrt{ }$ & \\
\hline Cambios de peso corporal & & $\checkmark$ & & $\checkmark$ & \\
\hline Alteración densidad mineral ósea & & $\checkmark$ & & $\sqrt{ }$ & \\
\hline Cambios músculo-esqueléticos & $\checkmark$ & & $\checkmark$ & $\checkmark$ & \\
\hline Daño función inmunológica & & $\checkmark$ & $\sqrt{ }$ & & $\checkmark$ \\
\hline Linfedema & $\checkmark$ & & $\checkmark$ & & \\
\hline Cambios gastrointestinales & & $\checkmark$ & & $\checkmark$ & $\checkmark$ \\
\hline
\end{tabular}

Con el fin de contrarrestar esos efectos colaterales ha surgido una importante línea de investigación en el área de la rehabilitación oncológica, que se enfoca en disminuir o evitar los efectos secundarios no deseados de la terapia antineoplásica a través del uso de la AF dirigida ${ }^{15}$.

Varias revisiones de ensayos clínicos aleatorios y metaanálisis han demostrado una asociación positiva entre AF durante y después de los tratamientos antineoplásicos, lo que se traduce en mejoría de la calidad de vida, funcionalidad, síntomas psicológicos y relacionados a la enfermedad y sus tratamientos ${ }^{16}$.

\section{Actividad física y cáncer de mama: bases biológicas}

Factores comunes que explican la relación entre AF y CM están principalmente basados en la modulación de marcadores inflamatorios, esteroides sexuales, eje insulina/factor de crecimiento insulino-símil (IGF-I) y adipokinas ${ }^{17}$ (Figura 1). La obesidad y el síndrome metabólico asociado reflejan la alteración de todos estos factores, siendo esta la condición corregible más relevante en la interacción AF-cáncer ${ }^{18}$.

La inflamación crónica es un factor de riesgo común para enfermedades cardiovasculares, síndrome metabólico, recurrencia y mortalidad por $\mathrm{CM}^{19}$. Ella se refleja en un aumento de citoquinas proinflamatorias, incluyendo factor de necrosis tumoral alfa (TNF $\alpha)$, interleukina 6 (IL6), leptina, adipoquinas y proteína $\mathrm{C}$ reactiva (PCR), junto a una disminución de citoquinas antiinflamatorias como la adiponectina ${ }^{20}$. Más de $50 \%$ de las pacientes con CM en tratamiento, y también sobrevivientes, tienen sobrepeso u obesidad, a las que frecuentemente se asocia, además, un bajo nivel de $\mathrm{AF}$, ambas condiciones unidas a un nivel crónico de inflamación ${ }^{21}$. Este estado inflamatorio crónico, en mujeres con $\mathrm{CM}$, puede persistir elevado incluso más allá de 5 años post-tratamiento ${ }^{19}$.

La resistencia a la insulina puede promover el desarrollo de CM por varias vías: aumento de glucosa disponible para células neoplásicas, hiperinsulinemia y consecuente aumento de los niveles disponibles de factor de crecimiento insulino-símil, este último estimulando la diferenciación, proliferación celular y suprimiendo la apoptosis. Además, la insulina puede aumentar la disponibilidad de estrógenos y andrógenos ${ }^{22}$.

Ballard-Barbash, en una revisión sistemática, analizó la relación entre AF y biomarcadores relevantes en CM que incluyeron: hormonas sexuales, 


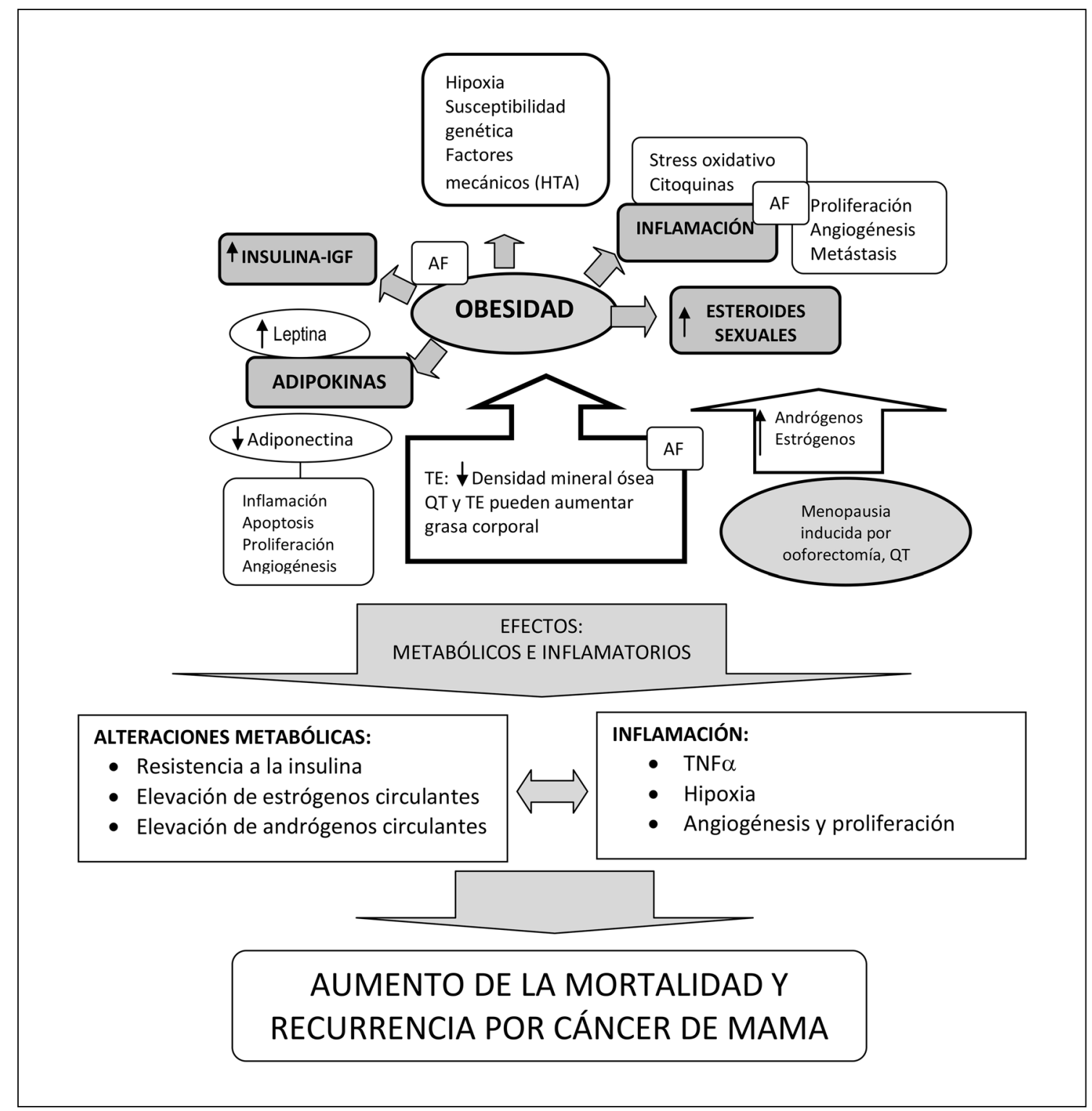

Figura 1. La actividad física (AF) interfiere favorablemente la relación entre cáncer y obesidad, síndrome metabólico e inflamación. (Adaptada de Sánchez C, et al. Obesidad y cáncer: la tormenta perfecta. Rev Med Chile 2014; 142 (2): 211-21). Abreviaciones figura: IGF: Factor de crecimiento insulino-símil. TNFa: Factor de necrosis tumoral alfa. HTA: Hipertensión arterial. QT: Quimioterapia. TE: Terapia endocrina. AF: Actividad física.

insulina, adipokinas y marcadores inflamatorios. En la mayoría de los estudios evaluados se demuestra que el ejercicio induce cambios en los niveles de todos estos biomarcadores ${ }^{23}$.

El estudio YALE es un estudio clínico aleatorizado controlado que evaluó el efecto de la $\mathrm{AF}$ supervisada sobre niveles de IL6, PCR y TNF- $\alpha$ en 75 mujeres posmenopáusicas sobrevivientes al CM. Mientras no se describieron cambios tras la intervención (AF por 6 meses), un análisis de subgrupos, no planificado, encontró que en aquellas mujeres que lograron cumplir más de $80 \%$ de los objetivos de la intervención, la IL6 se redujo significativamente ${ }^{17}$. 
El estudio HEAL (Health, Eating, Activity and Lifestyle), un estudio observacional en una cohorte de 1.183 pacientes con CM, evaluó el efecto del ejercicio sobre biomarcadores similares a los descritos en el estudio YALE. En esta cohorte, la AF fue asociada a bajos niveles de PCR y leptina, $\mathrm{y}$ altos niveles de IGF-I ${ }^{24}$.

Menores niveles de vitamina $\mathrm{D}$ en personas con menor actividad al aire libre, debido a menor estímulo de la luz ultravioleta sobre la piel, y a mayor depósito de vitamina $\mathrm{D}$ en el tejido graso, consecuentemente con menor biodisponibilidad de esta vitamina, también se han descrito como relevantes en la relación AF-cáncer ${ }^{25}$.

Dado los muchos elementos involucrados y la complejidad de los modelos de síndrome metabólico-obesidad-inflamación crónica, además de factores hormonales, es esperable que los estudios epidemiológicos sean inconsistentes en la búsqueda de un solo biomarcador ${ }^{26}$.

Junto a ello, y debido a la heterogeneidad clínica/molecular del CM, es posible que diferentes biomarcadores expliquen el riesgo de determinados tipos de CM (receptores de estrógenos positivos, receptores de estrógenos negativos, HER2 positivos) y que la AF interfiera en forma distinta en estos variados subtipos ${ }^{27}$.

\section{Actividad física en cáncer de mama}

Los beneficios de la AF para pacientes oncológicos son tanto fisiológicos como psicológicos. Hoy en día, se encuentran bien determinados $\mathrm{y}$ apoyados por evidencia que integra la $\mathrm{AF}$ en programas de intervención basados en ejercicios estructurados para pacientes, durante y después de los tratamientos antineoplásicos ${ }^{16}$.

El ejercicio se define como una AF realizada de manera dosificada y sistemática, es decir, con una frecuencia, intensidad, duración y modo específico, con propósito de mejorar variables de salud asociadas a la condición física. Para mejorar estas variables existen varios métodos de entrenamiento tales como aeróbico, resistencia y flexibilidad ${ }^{28}$.

\section{Prevención}

Si bien no hay demostración de que el sedentarismo aumente el riesgo de CM, sí existe una fuerte asociación entre AF y prevención. Un estudio prospectivo con más de 70.000 pacientes encontró que mujeres que mantenían AF regular presentaban menor riesgo de CM en $14 \%$ comparado con mujeres menos activas ${ }^{29}$.

De igual manera, una revisión de estudios epidemiológicos estimó que el riesgo de presentar $\mathrm{CM}$ se redujo en $25 \%$ en aquellas mujeres que eran físicamente activas, comparado con mujeres menos activas $^{30}$. Si bien la AF parece ser particularmente beneficiosa en mujeres postmenopáusicas, existe evidencia de que las menores de 45 años también logran reducir el riesgo ${ }^{31}$.

Dado el efecto paradójico del peso entre las pacientes pre y postmenopáusicas, la reducción del riesgo de CM por ejercicio probablemente no solo es mediada por el control del peso corporal ${ }^{32}$. El aumento en la AF parece disminuir el riesgo a través de la reducción de los estrógenos séricos y de los niveles de insulina y de IGF-1, como mencionamos previamente $\mathrm{e}^{33-35}$.

\section{Durante los tratamientos antineoplásicos}

El ejercicio físico en pacientes con $\mathrm{CM}$ es factible y seguro de realizar durante los tratamientos antineoplásicos sistémicos ${ }^{36,37}$.

Schmidt y cols., sugieren que el entrenamiento de resistencia es efectivo en el control de la fatiga y mejoría de la calidad de vida durante el tratamiento de quimioterapia, sin aumentar el riesgo de linfedema, ni dolor esperable por el ejercicio de resistencia ${ }^{12}$.

Van Waart y cols. encontraron que la concomitancia de quimioterapia y ejercicio (aeróbico más resistencia supervisado o de modo aeróbico en domicilio) induce mejoras en la funcionalidad física, mantiene el rendimiento cardiovascular, facilita la reincorporación precoz a la actividad laboral y se asocia a menor incidencia de náuseas, vómitos y dolor, comparado con un grupo sin ejercicio. Esto demuestra que un programa supervisado de ejercicio aeróbico y resistencia es efectivo; sin embargo, un programa de ejercicio para domicilio puede ser una alternativa viable ${ }^{13}$.

Vincent y cols., también evaluaron la efectividad de un programa de entrenamiento domiciliario, encontrando que los pacientes mejoraron el rendimiento cardiovascular con una adherencia cercana a $80 \%{ }^{36}$.

Courneya y cols. compararon ejercicio aeróbico, 30 y $60 \mathrm{~min}$, y aeróbico con resistencia, en mujeres con $\mathrm{CM}$ en quimioterapia, encontrando 
que la funcionalidad física mejoró con todos los tipos de entrenamiento. El entrenamiento aeróbico de mayor duración fue superior en parámetros como: calidad de vida, dolor y síntomas endocrinos. El ejercicio combinado logró mejores resultados en evaluaciones de fuerza muscular. El entrenamiento aeróbico de mayor duración fue superior al combinado, en evaluaciones de dolor y rendimiento aeróbico. Estos datos sugieren que sesiones de ejercicios de mayor volumen en tiempo son seguras, factibles de realizar y con efectos positivos en varios síntomas deletéreos para la paciente con $\mathrm{CM}^{37}$.

\section{En los sobrevivientes}

Para las sobrevivientes de CM, el ejercicio físico es también ampliamente recomendado y se ha vinculado no solo con mejorías en la calidad de vida, sino también con un aumento en la sobrevida ${ }^{38,39}$.

La ganancia de peso después del diagnóstico de CM es un efecto adverso común de los tratamientos antineoplásicos, acentuado en sobrevivientes que presentan bajos niveles de $\mathrm{AF}^{40}$. Pacientes con sobrepeso u obesidad al momento del diagnóstico o con ganancias de peso importante durante el tratamiento presentan menores tasas de sobrevida ${ }^{41}$. El ejercicio físico modularía el nivel de estróge$\operatorname{nos}^{42}$, disminuiría la resistencia a la insulina y la hiperinsulinemia ${ }^{43}$, factores que explicarían una mejoría en la sobrevida.

Una revisión sistemática, que incluyó 3.700 pacientes, analizó el efecto de distintos programas de AF en la calidad de vida de sobrevivientes de cáncer, encontrando que el efecto fue positivo en distintos dominios de la calidad de vida relacionada a la salud y fatiga, confirmando un efecto positivo de la incorporación de AF en esta población $^{38}$. Un estudio de una cohorte prospectiva de casi 3.000 sobrevivientes de CM sugiere que niveles altos de AF se asocian a una disminución del riesgo de recurrencia y mortalidad específica por la enfermedad. El mayor beneficio se observó en las mujeres que caminaban entre 3 y 5 h por semana $^{39}$.

\section{Prescripción del ejercicio}

La prescripción de ejercicio en pacientes con $\mathrm{CM}$ debe ser individualizada, tomando en cuen- ta tanto los efectos del cáncer y del tratamiento en la capacidad funcional, considerando modo, intensidad, duración, frecuencia y progresión del ejercicio $^{44-46}$.

La mayoría de los estudios han evaluado 5 a 12 semanas de ejercicio (durante los tratamientos antineoplásicos), 3 a 5 veces por semana, incorporando ejercicios de resistencia y aeróbicos de moderada a vigorosa intensidad por 30 a $60 \mathrm{~min}$ $y$, generalmente, utilizando la marcha como ejercicio $^{38}$. La marcha es conveniente para muchos pacientes sin tener en cuenta edad o estado de enfermedad; se puede continuar a través de las distintas fases de la enfermedad y no requiere de recursos costosos o especializados ${ }^{44,46}$.

No obstante, el ejercicio físico tiene riesgos inherentes. La evidencia ha reportado escasas complicaciones en pacientes oncológicos sometidos a programas de actividad física supervisada, porque los criterios de exclusión en los ensayos clínicos descartan a los pacientes susceptibles de desarrollar eventos adversos, tales como aquellos con patología cardiopulmonar sin control médico, caquexia severa, metástasis óseas, entre otras ${ }^{16}$. Es por ello que, previo a su prescripción, es necesaria la evaluación específica de posibles factores de riesgo o antecedentes cardiopulmonares y neurológicos. Se debe tener especial cuidado en pacientes que estén recibiendo quimioterapia con probables efectos colaterales a nivel cardio-pulmonar, y en aquellos que presentan etapas avanzadas de la enfermedad ${ }^{44-46}$ (Tabla 3 ).

En las pacientes con CM sometidas a cirugía, especialmente con disección y/o radioterapia axilar, existe el temor de que el ejercicio sea un desencadenante de linfedema en el brazo ipsilateral, el cual se desarrolla cuando la carga linfática excede la capacidad de transporte linfático ${ }^{47}$. Algunos estudios han usado ejercicio de resistencia en sus intervenciones, encontrando que las pacientes no desarrollaron ni empeoraron, en el caso que corresponda, linfedema de la extremidad ${ }^{11,12}$.

Además, se recomienda que todo programa de AF sea dirigido y supervisado por un profesional del área de la rehabilitación, con experiencia y formación en el área oncológica y ciencias de la actividad física, para monitorear la respuesta al ejercicio y de este modo regular y ajustar el programa según el estado físico o emocional de cada paciente ${ }^{46,48}$ (Tabla 4 ). 
Tabla 3. Consideraciones para interrumpir temporal o definitivamente programas de actividad fïsica

\begin{tabular}{ll} 
Complicación & Consideración \\
\hline Plaquetas $<50.000 / \mathrm{mm}^{3}$, Hemoglobina $<8 \mathrm{~g} / \mathrm{dl}$ & Evitar actividad física de alta intensidad o impacto \\
\hline Leucocitos $<1.500 / \mathrm{mm}^{3}$ & Evitar actividad física que aumenten riesgo de infección (natación) \\
\hline Neurotoxicidad mayor a grado 2 & Evitar actividad física que requiera equilibrio y coordinación (treadmill) \\
Metástasis ósea sintomática o asintomática & Evitar actividad física de alto impacto \\
\hline Fatiga severa & Iniciar actividad física de baja intensidad \\
\hline Fiebre $\left(\mathrm{T}^{\circ}\right.$ corporal mayor a $38^{\circ} \mathrm{C}$ axilar) & No realizar actividad física \\
\hline
\end{tabular}

Tabla 4. Recomendaciones de actividad física en mujeres con diagnóstico de cáncer de mama y sobrevivientes

\begin{tabular}{|c|c|c|c|}
\hline & Aeróbico & Resistencia & Flexibilidad \\
\hline Dosis & $\begin{array}{l}150 \mathrm{~min} / \mathrm{semana} \text { de intensidad } \\
\text { moderada o } 75 \mathrm{~min} / \mathrm{semana} \text { de } \\
\text { intensidad vigorosa }\end{array}$ & $\begin{array}{l}2 \text { sets de } 8 \text { a } 12 \text { repeticiones para } \\
\text { cada ejercicio con } 1 \mathrm{~min} \text { de reposo } \\
\text { entre cada set }\end{array}$ & $\begin{array}{l}\text { Elongar grandes } \\
\text { grupos musculares y } \\
\text { tendones }\end{array}$ \\
\hline Intensidad & $\begin{array}{l}60 \text { a } 80 \% \text { frecuencia cardiaca } \\
\text { máxima teórica }\end{array}$ & 50-70\% de una repetición máxima & $\begin{array}{l}10-30 \text { segundos por } \\
\text { grupos musculares }\end{array}$ \\
\hline Frecuencia & $\begin{array}{l}3 \text { veces a la semana o a diario con } \\
\text { ejercicio intermitente en pacientes } \\
\text { desacondicionados }\end{array}$ & 2 veces a la semana & \\
\hline Tipo & $\begin{array}{l}\text { Marcha, bicicleta, elíptica, natación, } \\
\text { tenis }\end{array}$ & $\begin{array}{l}\text { Ejercicios de grandes grupos } \\
\text { musculares }\end{array}$ & \\
\hline $\begin{array}{l}\text { Consideraciones } \\
\text { especiales }\end{array}$ & $\begin{array}{l}\text { En pacientes desacondicionados se } \\
\text { sugiere entrenamiento diario con } \\
\text { ejercicio intermitente ( } 3 \text { veces al día } \\
\text { por } 10 \mathrm{~min} \text { ) }\end{array}$ & $\begin{array}{l}\text { Siempre con supervisión. Iniciar con } \\
\text { cargas bajas. No hay límite superior } \\
\text { en la carga de peso para las sobre- } \\
\text { vivientes; sin embargo, se deben } \\
\text { controlar los síntomas, incluyendo } \\
\text { el linfedema }\end{array}$ & \\
\hline $\begin{array}{l}\text { Contraindicaciones } \\
\text { para iniciar un } \\
\text { programa de ejercicio }\end{array}$ & $\begin{array}{l}\text { Mujeres con molestias en el brazo } \\
\text { u hombro post cirugía (dificultad } \\
\text { para levantar el brazo, disminución } \\
\text { del rango de movimiento, } \\
\text { aumento de volumen) deben } \\
\text { buscar resolver la condición antes } \\
\text { de iniciar ejercicio. }\end{array}$ & & \\
\hline
\end{tabular}

\section{Traslación del conocimiento a la práctica clínica}

Lograr adherencia de los pacientes oncológicos a programas de AF dirigida es un desafío ${ }^{16}$, y específicamente, en el caso de pacientes con CM recibiendo quimioterapia, el desafío puede ser mayor, principalmente por los efectos secundarios al tratamiento ${ }^{49}$.
Sólo un tercio de los pacientes potencialmente elegibles para participar en ensayos clínicos acepta participar ${ }^{16,49}$. Una de las razones más reportadas es la creencia de que el manejo contra el cáncer requiere de reposo físico ${ }^{16}$. Además, se describen otras barreras relacionadas a síntomas físicos que limitan la AF, tanto para aceptar participar como para asistir a las sesiones programadas (tales como vómitos, náuseas). Otras limitantes se relacionan 
Tabla 5. Un ejemplo de indicación de actividad física aeróbica para pacientes con cáncer de mama en quimioterapia

\begin{tabular}{|lll|}
\hline & Semana $\mathbf{1}$ a $\mathbf{6}$ & Semana $\mathbf{6}$ a $\mathbf{1 2}$ \\
\hline Intensidad & FCent=(FCMt-FCrep)x(60\%)+FCrep) & FCent=(FCMt-FCrep)x(80\%)+FCrep) \\
& Borg 11 & Borg 13 \\
\hline Frecuencia & 3 veces a la semana (50 mins) & 3 veces a la semana (60 mins) \\
\hline Tipo & Bicicleta estática o marcha & Bicicleta estática o marcha \\
Evaluar progresión de la capacidad física* & Antes de iniciar programa y en semana 6 & Semana 12 \\
\hline
\end{tabular}

FCent: frecuencia cardiaca de entrenamiento. FCMt: frecuencia cardiaca máxima teórica. FCrep: frecuencia cardiaca de reposo. Borg: Escala Borg de esfuerzo percibido (Borg. G. Las bases psicofísicas del esfuerzo percibido. J. Med. Sci. Sports Exercise, v. 14, n. 5, p. 377-381, 1982). *Utilizar una prueba de capacidad física (ejemplos: test de marcha 6 min, timed up and go).

a la situación personal de cada individuo, en relación a su estado financiero, transporte, cuidado de hijos, falta de tiempo, etc; estado psicológico y secuelas estéticas secundarias a los tratamientos antineoplásicos, como la cirugía o el tratamiento hormonal (amputación, aumento de peso, entre otros). Por último, está la barrera motivacional, falta de interés o de información de que la AF dirigida tiene siempre la opción de adaptarse específicamente a cada paciente ${ }^{16,49}$. A pesar de todo esto, una vez que el paciente adhiere a los programas de entrenamiento, más de $80 \%$ se mantendrá activo en el programa bajo supervisión ${ }^{16}$.

Basados en el conocimiento adquirido en esta revisión, proponemos un ejemplo de indicación de actividad física aeróbica dirigido a la práctica clínica (Tabla 5).

\section{Conclusión}

La AF, indicada de manera dirigida y supervisada, generaría efectos positivos, emocionales y físicos, en pacientes con CM, y su realización es factible incluso cuando las pacientes están recibiendo tratamientos antineoplásicos, específicamente la quimioterapia.

El conocimiento de la heterogeneidad del CM y de los mecanismos biológicos relacionados a la relación ejercicio-cáncer permitirán personalizar la indicación de AF y determinar modelos que evalúen su impacto en la calidad de vida y en el pronóstico de la enfermedad.

Es fundamental desarrollar políticas públicas en salud para aumentar la participación de los pacientes oncológicos en programas de AF dirigida, a través de entrega de información adecuada y oportuna, analizar las necesidades y las barreras de cada población oncológica según el centro en el que recibe el tratamiento contra el cáncer y determinar el interés de los pacientes por participar en programas de ejercicio. Además, es importante educar a otros profesionales de la salud que se relacionan con los pacientes para que influyan de manera positiva en la participación de los pacientes en AF supervisada.

\section{Referencias}

1. http://www.cancerresearchuk.org/cancer-info/cancerstats/world/the-global-picture/. (Fecha de acceso 28 de agosto de 2012).

2. http://www.ispch.cl/sites/default/files/Actualizacion_ Programa_Nac_de_Cancer.pdf

3. Anand P, Kunnumakkara AB, Sundaram C, Harikumar $\mathrm{KB}$, Tharakan ST, Lai OS, et al. Cancer is a preventable disease that requires major lifestyle changes. Pharm Res 2008; 25 (9): 2097-116.

4. Desantis C, Ma J, Bryan L, Jemal A. Breast Cancer Statistics, 2013. CA Cancer J Clin 2014; 64 (1): 52-62.

5. Lenihan DJ, Cardinale DM. Late cardiac effects of cancer treatment. J Clin Oncol 2012; 30 (30): 3657-64.

6. Petrelli F, Borgonovo K, Cabiddu M, Lonati V, Barni S. Mortality, leukemic risk, and cardiovascular toxicity of adjuvant anthracycline and taxane chemotherapy in breast cancer: a meta-analysis. Breast Cancer Res Treat 2012; 135 (2): 335-46.

7. Stull V, Snyder D, Demark-Wahnefried W. Lifestyle interventions in cancer survivors: designing programs that meet the needs of this vulnerable and growing population. J Nutr 2007; 137 (1): 243S-248S.

8. Courneya K, Lee J, Fairey A, Campbell K, Ladha A, Friedenreich $\mathrm{C}$, et al. Physical activity in cancer survi- 
vors: implications for recurrence and mortality. Cancer Therapy 2004; 2: 1-12.

9. Cleeland C, Allen J, Roberts S, Brell J, Giralt S, Khakoo A, et al. Reducing the toxicity of cancer therapy: recognizing needs, taking action. Nat Rev Clin Oncol 2012; 9 (8): 471-8.

10. Goldhirsch A, Winer E, Coates A, Gelber R, Piccart-Gebhart $M$, Thürlimann B, et al. Personalizing the treatment of women with early breast cancer: highlights of the St Gallen International Expert Consensus on the Primary Therapy of Early Breast Cancer 2013. Ann Oncol 2013; 24 (9): 2206-23.

11. Courneya K, Segal R, Mackey J, Gelmon K, Reid R, Friedenreich C, et al. Effects of Aerobic and Resistance Exercise in Breast Cancer Patients Receiving Adjuvant Chemotherapy: A Multicenter Randomized Controlled Trial. Journal of Clinical Oncology 2007; 25 (28): 4396404.

12. Schmidt M, Wiskemann J, Armbrust P, Schneeweiss A, Ulrich C, Steindorf K. Effects of resistance exercise on fatigue and quality of life in breast cancer patients undergoing adjuvant chemotherapy: A randomized controlled trial. International Journal of Cancer 2015; 137 (2): 471-80.

13. Van Waart H, Stuiver M, van Harten W, Geleijn E, Kieffer J, Buffart L, et al. Effect of Low-Intensity Physical Activity and Moderate- to High-Intensity Physical Exercise During Adjuvant Chemotherapy on Physical Fitness, Fatigue, and Chemotherapy Completion Rates: Results of the PACES Randomized Clinical Trial. Journal of Clinical Oncology 2015; 33 (17): 1918-27.

14. Courneya K, Mackey J, McKenzie D. Exercise for breast cancer survivors: research evidence and clinical guidelines. Phys Sportsmed 2002; 30 (8): 33-42.

15. Courneya KS. Exercise in cancer survivors: an overview of research. Med Sci Sports Exerc 2003; 35 (11): 184652.

16. Bouillet T, Bigard X, Brami C, Chouahnia K, Copel L, et al. Role of physical activity and sport in oncology: Scientific commission of the National Federation Sport and Cancer CAMI. Critical Reviews in Oncology/hematology 2015; 94 (1): 74-86.

17. Jones S, Thomas G, Hesselsweet S, Álvarez-Reeves M, Yu H, Irwin M. Effect of exercise on markers of inflammation in breast cancer survivors: The yale exercise and survivorship study. Cancer Prevention Research 2013; 6 (2): 109-18.

18. Lynch BM. Sedentary Behavior and Cancer: A Systematic Review of the Literature and Proposed Biological Mechanisms. Cancer Epidemiol Biomarkers Prev 2010; 19 (11): 2691-709.
19. Iyengar N, Hudis C, Dannenberg A. Obesity and inflammation: new insights into breast cancer development and progression. Am Soc Clin Oncol Educ Book 2013. p. 46-51.

20. Roxburgh C, McMillan D. Cancer and systemic inflammation: treat the tumour and treat the host. Br J Cancer 2014; 110 (6): 1409-12.

21. Sánchez C, Ibáñez C, Klaassen J. [The link between obesity and cancer]. Rev Med Chile 2014; 142 (2): 211-21.

22. Simpson E, Brown K. Obesity and breast cancer: Role of inflammation and aromatase. J Mol Endocrinol 2013; 51 (3): T51-9.

23. Ballard-Barbash R, Friedenreich C, Courneya K, Siddiqi S, McTiernan A, Alfano C. J Natl Cancer Inst 2012; 104 (11): 815-40.

24. Stover A, Reeve B, Piper B, Alfano C, Smith A, Mitchell $S$, et al. Deriving clinically meaningful cut-scores for fatigue in a cohort of breast cancer survivors: a Health, Eating, Activity, and Lifestyle (HEAL) Study. Qual Life Res. Netherlands 2013; 22 (9): 2279-92.

25. Chlebowski RT. Vitamin D and breast cancer incidence and outcome. Anticancer Agents Med Chem 2013; 13 (1): 98-106.

26. Neilson H, Friedenreich C, Brockton N, Millikan R. Physical activity and postmenopausal breast cancer: Proposed biologic mechanisms and areas for future research. Cancer Epidemiology Biomarkers and Prevention. 2009. p. 11-27.

27. Ligibel J. Lifestyle factors in cancer survivorship. J Clin Oncol 2012; 30 (30): 3697-704.

28. Bouchard C, Shepard R, Stephens T. Physical activity, fitness and health. International proceedings and concensus statement. Champaign (IL): Human Kinetics, 1994: 1055.

29. McTiernan A, Kooperberg C, White E, Wilcox S, Coates R, Adams-Campbell LL, et al. Recreational Physical Activity and the Risk of Breast Cancer in Postmenopausal Women. JAMA 2003; 290 (10): 1331.

30. Lynch B, Neilson H, Friedenreich C. Physical activity and breast cancer prevention. Recent Results Cancer Res 2011; 186: 13-42.

31. Thune I, Brenn T, Lund E, Gaard M. Physical Activity and the Risk of Breast Cancer. N Engl J Med 1997; 336 (18): 1269-75.

32. Nelson H, Zakher B, Cantor A, Fu R, Griffin J, O'meara E, et al. Risk Factors for Breast Cancer for Women Aged 40 to 49 Years A Systematic Review and Meta-analysis. Ann Intern Med 2012; 156 (9): 635-48.

33. Irwin ML, Varma $\mathrm{K}$, Álvarez-Reeves $\mathrm{M}$, Cadmus $\mathrm{L}$, Wiley A, Chung GG, et al. Randomized Controlled Trial of Aerobic Exercise on Insulin and Insulin-like Growth 
Factors in Breast Cancer Survivors: The Yale Exercise and Survivorship Study. Cancer Epidemiol Biomarkers Prev 2009; 18 (1): 306-13.

34. Ligibel J, Campbell N, Partridge A, Chen W, Salinardi $\mathrm{T}$, Chen $\mathrm{H}$, et al. Impact of a Mixed Strength and Endurance Exercise Intervention on Insulin Levels in Breast Cancer Survivors. J Clin Oncol 2008; 26 (6): 907-12.

35. Fairey A, Courneya K, Field C, Bell G, Jones L, Mackey $\mathrm{J}$. Effects of exercise training on fasting insulin, insulin resistance, insulin-like growth factors, and insulin-like growth factor binding proteins in postmenopausal breast cancer survivors: a randomized controlled trial. Cancer Epidemiol Biomarkers Prev 2003; 12 (8): 721-7.

36. Vincent F, Labourey L, Leobon S, Antonini T, Lavau $S$, Tubiana N. Effects of a home based walking training program on cardiorespiratory fitness in breast cancer patients receiving adjuvant chemotherapy: a pilot study. Eur J Physi Rehabil Med 2013; 49 (3): 319-29.

37. Courneya K, McKenzie D, Mackey J, Gelmon K, Friedenreich C, Yasui Y, Segal R. Effects of Exercise Dose and Type During Breast Cancer Chemotherapy: Multicenter Randomized Trial. JNCI Journal of the National Cancer Institute 2013; 105 (23): 1821-32.

38. Mishra S, Scherer R, Snyder C, Geigle P, Gotay C. Are exercise programs effective for improving health related quality of life among cancer survivors? A systematic review and meta-analysis. Oncol Nurs Forum 2014; 41 (6): E326-42.

39. Holmes M, Chen W, Feskanich D, Kroenke C, Colditz G. Physical Activity and Survival After Breast Cancer Diagnosis JAMA 2015; 293 (20): 2479-86.

40. Demark-Wahnefried W, Winer E, Rimer B. Why women gain weight with adjuvant chemotherapy for breast cancer. J Clin Oncol 1993; 11 (7): 1418-29.

41. Obermair A, Kurz C, Hanzal E, et al. The influence of obesity on the disease-free survival in primary breast cancer. Anticancer Res 1995; 15 (5B): 2265-9.

42. Holmberg L, Norden T, Lindgren A, Wide L, Degerman M, Adami HO. Pre-operative oestradiol levels-relation to survival in breast cancer. Eur J Surg Oncol 2001; 27 (2): 152-6.

43. Goodwin PJ, Ennis M, Pritchard KI, et al. Fasting insulin and outcome in early-stage breast cancer: results of a prospective cohort study. J Clin Oncol 2002; 20 (1): 4251.

44. Winningham M. Therapeutic exercise: guidelines and precautions. In: Winningham M, Barton-Burke M, editors. Fatigue in Cancer: a multidimensional approach. Sudbury M: Jones \& Barlett 2000; 339-50.

45. Schmitz K, Courneya K, Matthews C, Demark-Wahnefried W, Galvao D, Pinto B, et al. American college of sports medicine roundtable on exercise guidelines for cancer survivors. Medicine and Science in Sports and Exercise 2010; 42 (7): 1409-26. http://doi.org/10.1249/ MSS.0b013e3181e0c112.

46. Wolin KY, Schwartz AL, Matthews CE, Courneya KS, Schmitz KH. Implementing the Exercise Guidelines for Cancer Survivors. Journal of Supportive Oncology 2012; 10 (5): 171-7. http://doi.org/10.1016/j. suponc.2012.02.001.

47. Földi E, Földi M, Clodius L: "The Lymphedema Chaos: A lancet” Annals Plastic Surg 1989; 22 (6): 505-15.

48. Watson T, Mock V. Exercise as an Intervention for Cancer-Related Fatigue. Physical Therapy 2004; 84 (8): 736-43.

49. Courneya K, Segal R, Gelmon K, Mackey J, Friedenreich $\mathrm{C}$, et al. Predictors of adherence to different types and doses of supervised exercise during breast cancer chemotherapy. The International Journal of Behavioral Nutrition and Physical Activity 2014; 11 (1): 85. 